\title{
Concepções de docentes e discentes de Gestão Hospitalar sobre a segurança do paciente
}

\author{
Ricardo Mendes de Souza \\ Discente do Curso Superior de Tecnologia em Gestão Hospitalar, Escola de Saúde da Universidade \\ Federal do Rio Grande do Norte, Natal, RN, Brasil \\ 凶ricardo-cadu@outlook.com
}

\section{Rildécio Medeiros Filho}

Discente do Curso Superior de Tecnologia em Gestão Hospitalar, Escola de Saúde da Universidade Federal do Rio Grande do Norte, Natal, RN, Brasil

\section{Darlyane de Lima e Silva}

Tecnóloga em Gestão Hospitalar, Escola de Saúde da Universidade Federal do Rio Grande do Norte, Natal, RN, Brasil

\section{Cláudia Cristiane Filgueira Martins Rodrigues}

Docente da Escola de Saúde da Universidade Federal do Rio Grande do Norte, Natal, RN, Brasil

\section{Pétala Tuani Candido de Oliveira Salvador}

Docente da Escola de Saúde da Universidade Federal do Rio Grande do Norte, Natal, RN, Brasil

\section{Resumo:}

O Programa Nacional de Segurança do Paciente prioriza em suas diretrizes a inclusão do tema nos currículos dos cursos de formação em saúde de nível técnico, superior e de pós-graduação, além da promoção de capacitação continuada para gestores e profissionais de saúde em segurança do paciente. Neste estudo, destaca-se a relevância desta reflexão no âmbito dos cursos de formação de gestores em saúde como mecanismo de subsidiar a profissionalização de gestores qualificados com a compreensão sobre qualidade em saúde e segurança do paciente. Essa pesquisa objetivou desvelar a concepção de docentes e discentes de Gestão Hospitalar sobre a segurança do paciente. Trata-se de estudo descritivo, transversal, de abordagem qualitativa, com a participação de dezenove docentes e doze discentes. Os dados decorrentes dos questionários de caracterização foram tabulados e analisados a partir de estatística descritiva simples. Já os dados textuais provenientes das entrevistas foram transcritos e analisados com suporte do software Interface de $R$ pour Analyses Multidimensionnelles de Textes et de Questionneires (IRAMUTEQ). Foram analisadas seis classes que emergiram das entrevistas com os sujeitos de pesquisa, cinco acerca das ações para a segurança do paciente - trabalho em equipe, processo de cuidado, cuidado multiprofissional, integração da gestão e cultura de segurança - e uma sobre as concepções sobre a segurança do paciente. Concluiu-se que a segurança do paciente deve perpassar por todos os profissionais, especialmente o gestor, que planejará recursos para que essas ações aconteçam. Assim, como estratégia, apresenta-se a capacitação dos gestores em saúde como caminho para fortalecer a cultura de segurança.

Palavras-chave: Ciências da saúde, segurança do paciente, gestão hospitalar, gestão da qualidade es. 


\title{
Hospital management teacher and student conceptions on patient safety
}

\begin{abstract}
:
The National Patient Safety Program prioritizes in its guidelines the inclusion of the theme in the curricula of health training courses at technical, higher, and graduate level, in addition to promoting continued training for managers and professionals of patient safety. In this study, we highlight the relevance of this reflection in the context of training courses for health managers as a mechanism to support the professionalization of qualified managers with an understanding of quality in patient health and safety. This research aimed to unveil the concept of teachers and students of the Higher Course of Technology in Hospital Management of the School of Health of the Federal University of Rio Grande do Norte, about patient safety. This is a descriptive, crosssectional study with a qualitative approach, with the participation of nineteen teachers and twelve students. Data from the characterization questionnaires were tabulated and analyzed using simple descriptive statistics. The textual data from the interviews were transcribed and analyzed with the support of the R interface for Multidimensional Analysis of Textes and Questionneires (IRAMUTEQ). Six classes that emerged from the interviews with the research subjects were analyzed, five about the actions for patient safety - teamwork, care process, multiprofessional care, integration of management and safety culture - and one on the conceptions about patient safety. It was concluded that patient safety should be permeated by all professionals, especially the manager, who will plan resources for these actions to happen. Thus, as a strategy, the training of health managers is presented to strengthen the safety culture.
\end{abstract}

Keywords: Science health, patient safety, hospital management, quality management.

\section{Concepciones de profesores y estudiantes de gestión hospitalaria sobre la seguridad del paciente}

\section{Resumen:}

El Programa Nacional de Seguridad del Paciente prioriza en sus directrices la inclusión del tema en los planes de estudios de los cursos de formación en salud a nivel técnico, superior y de posgrado, además de promover la formación continua para gerentes y profesionales de seguridad del paciente. En este estudio, destacamos la relevancia de esta reflexión en el contexto de cursos de formación para gestores de salud como mecanismo de apoyo a la profesionalización de gestores cualificados con una comprensión de la calidad en la salud y seguridad del paciente. Esta investigación tuvo como objetivo revelar la concepción de los maestros y estudiantes de Gestión Hospitalaria sobre la seguridad del paciente. Este es un estudio descriptivo, transversal con un enfoque cualitativo, con la participación de diecinueve maestros y doce estudiantes. Los datos resultantes de los cuestionarios de caracterización se tabularon y analizaron utilizando estadísticas descriptivas simples. Los datos textuales de las entrevistas, por otro lado, se transcribieron y analizaron utilizando el software Interface de $\mathrm{R}$ pour Analyzes Multidimensionnelles de Textes et de Questionneires (IRAMUTEQ). Se analizaron seis clases que surgieron de las entrevistas con los sujetos de investigación, cinco sobre las acciones para la seguridad del paciente - trabajo en equipo, proceso de cuidado, atención multiprofesional, integración de la gestión y la cultura de seguridad - y una sobre las concepciones sobre la seguridad del paciente. Se concluyó que la seguridad del paciente debe ser permeada por todos los profesionales, especialmente el gerente, quien planificará los recursos para que estas acciones sucedan. Así, como estrategia, la formación de los gestores de salud se presenta como una forma de fortalecer la cultura de seguridad.

Palabras clave: Ciencias de la salud, seguridad del paciente, gestión hospitalaria, gestión de la calidad.

\section{INTRODUÇÃO}

A busca pela qualidade em saúde em instituições de saúde, sejam públicas ou privadas, têm sido cada vez mais discutida com o objetivo de dar credibilidade à assistência. No entanto, para obter qualidade, apenas exercer as atividades no ambiente de saúde da melhor forma possível já não é suficiente (BALSANELLI; JERICÓ, 2005). 
Nos dias atuais, a busca pela qualidade tornou-se prioridade nas organizações de saúde. Deste modo, a gestão da qualidade foi atrelada à análise com base na melhoria contínua da estrutura, dos processos e resultados, uma proposta criada por Avedis Donabedian (DONABEDIAN, 1980; GOMES et al., 2019).

Constatou-se, com isso, a necessidade da segurança do paciente como indicador para avaliação da qualidade assistencial (VITURI; ÉVORA, 2015). A segurança do paciente, que atualmente é compreendida como a redução do risco de dano desnecessário associado ao cuidado de saúde a um mínimo aceitável (BRASIL, 2013), tornou-se imprescindível para o aperfeiçoamento da qualidade nos serviços de saúde (MINUZZ; SALUM; LOCKS, 2016).

No Brasil, as ações propostas aconteceram com a criação do Programa Nacional de Segurança do Paciente (PNSP), pela Portaria No 529 de 2013, que estabeleceu objetivos, definições e estratégias para implementação do cuidado seguro nos serviços de saúde (BRASIL, 2013).

Neste contexto, é notável que a participação da equipe multidisciplinar é essencial para efetivação das ações propostas. Assim, é importante conhecer a percepção de qualidade dos profissionais para melhor direcionar as estratégias e ações, atender às expectativas e propiciar uma tomada de decisão compatível com a instituição de saúde (SILVA; RODOVALHO, 2012).

Nesse ínterim, a cultura de segurança tornou-se um componente estrutural nos serviços de saúde, importante na diminuição de incidentes (ANDRADE et al., 2016). Atualmente, a cultura de segurança é definida como o produto de valores, atitudes, competências e padrões de comportamento individuais e de grupo, os quais determinam o compromisso, o estilo e proficiência da administração de uma organização saudável e segura (HEALTH AND SAFETY COMMISSION, 1993; REIS, 2013).

Sendo assim, o apoio da alta gestão hospitalar se tornou necessário para a promoção da cultura de segurança. No entanto, estudos recentes apontam que a gestão hospitalar ainda é ausente quando as questões envolvem a segurança do paciente, o que implica em uma cultura desfavorável e no desestímulo dos profissionais de saúde que atuam na linha de cuidado (KAWAMOTO et al., 2016). 
Portanto, a inserção da temática durante a formação acadêmica dos profissionais da saúde tornou-se imprescindível. Assim sendo, o PNSP prioriza em suas diretrizes a inclusão do tema nos currículos dos cursos de formação em saúde de nível técnico, superior e de pósgraduação, além da promoção de capacitação continuada para gestores e profissionais de saúde em segurança do paciente (BRASIL, 2013).

O gestor precisa ser educado sobre a importância da cultura de segurança. Sobre isso, a National Patient Safety Foundation, em 2015, recomendou como tática para manter e estabelecer uma cultura de segurança que os conselhos, gestores e reguladores em saúde tenham educação suficiente sobre os fundamentos de ciência de segurança do paciente para incentivar as iniciativas culturais (NATIONAL PATIENT SAFETY FOUNDATION, 2015).

Compreende-se, dessa forma, que é vital abordar a temática no contexto da formação de gestores hospitalares. Neste estudo, destaca-se a relevância desta reflexão no âmbito dos cursos de formação de gestores em saúde, como mecanismo de subsidiar a profissionalização de gestores qualificados com a compreensão sobre qualidade em saúde e segurança do paciente.

Com isso, apresenta-se a questão desse estudo: qual a concepção de docentes e discentes de Gestão Hospitalar sobre a segurança do paciente? Objetiva-se desvelar a concepção de docentes e discentes de Gestão Hospitalar sobre a segurança do paciente.

\section{MATERIAL E MÉTODOS}

Trata-se de estudo descritivo, transversal, de abordagem qualitativa. A pesquisa foi desenvolvida no âmbito da Escola de Saúde da Universidade Federal do Rio Grande do Norte (ESUFRN), Unidade Acadêmica Especializada em Educação Profissional em Saúde, que oferta cursos da área da saúde nos níveis técnico, de graduação e de pós-graduação (ESUFRN, 2018).

Dentre tais cursos, a pesquisa teve como campo de investigação o Curso Superior de Tecnologia em Gestão Hospitalar, graduação tecnológica com uma carga horária total de 2440 horas distribuídas em seis períodos. 
O Tecnólogo em Gestão Hospitalar possui o seguinte campo de atuação proposto: atua no planejamento, organização e gerenciamento dos processos de trabalho em saúde, envolvendo a área de gestão de pessoas, materiais e equipamentos; organiza e controla compras e custos, áreas de apoio e logística hospitalar, bem como acompanha e supervisiona contratos e convênios, através dos princípios da gestão, qualidade e viabilidade dos serviços presta suporte aos setores fins; e pode atuar em hospitais - e seus setores, clínicas e unidades de saúde, laboratórios médicos e empresas prestadoras de serviço em saúde (ESUFRN, 2018).

A população de estudo foram os docentes e discentes do Curso Superior de Tecnologia em Gestão Hospitalar da ESUFRN. A amostra foi do tipo intencional e incluiu todos os docentes que atuam no curso e os discentes concluintes do mesmo no semestre 2019.1.

Neste semestre, o Curso contava com 25 docentes efetivos atuantes e 25 discentes concluintes. Estes quantitativos, então, constituíram a população da pesquisa - 50 sujeitos, no total.

Para os docentes, foram estabelecidos como critérios de inclusão: ser docente efetivo e ministrar aulas no Curso Superior de Tecnologia em Gestão Hospitalar da ESUFRN. Foram excluídos os docentes afastados por qualquer motivo. A amostra totalizou 19 docentes.

Quanto aos discentes, foram incluídos aqueles matriculados nos módulos correspondentes ao sexto período do curso, considerados, portanto, concluintes. Foram excluídos os discentes desnivelados, que estivessem com módulos a cursar dos períodos anteriores do curso. Participaram da pesquisa 11 discentes.

A coleta de dados foi realizada a partir de entrevista nos meses de setembro a novembro com docentes e junho com os discentes. A entrevista com os professores ocorreu de forma individual, em horário e local previamente combinado, a fim de garantir o mapeamento das percepções e vivências de todos os docentes do curso, e teve seu áudio gravado conforme aquiescência dos participantes.

Para os discentes, inicialmente, foi pensada a realização de um grupo focal. No entanto, a partir da recomendação de que os grupos focais devem ter, para garantir sua eficácia, a participação de 6 a 15 pessoas (MINAYO, 2010), não foi possível prosseguir porque não havia o mínimo de estudantes para continuar a entrevista no dia agendado para tal. Desse 
modo, optou-se por realizar a entrevista individual com os discentes que haviam comparecido e encaminhar o formulário eletrônico aos demais.

Tanto no âmbito das entrevistas individuais com os docentes e discentes, como no formulário eletrônico com estudantes, foram utilizados dois instrumentos de pesquisa: 1) questionário de caracterização; e 2) roteiro de realização da entrevista, composto por quatro questões sobre compreensão acerca da temática segurança do paciente, ações associadas, módulos em que foram ministrados aspectos da segurança do paciente no Curso de Gestão Hospitalar e a importância do gestor hospitalar no âmbito da segurança do paciente.

Os dados decorrentes dos questionários de caracterização foram tabulados e analisados a partir de estatística descritiva simples. Já os dados textuais provenientes das entrevistas foram transcritos e analisados com suporte do software Interface de R pour Analyses Multidimensionnelles de Textes et de Questionneires (IRAMUTEQ), o qual processa análises lexicais de dados textuais ao fornecer contextos e classes por meio do julgamento da semelhança de seus vocabulários, de maneira a contribuir na compreensão do ambiente de sentido das palavras, logo, indicar elementos das representações referentes ao objeto estudado (CAMARGO; JUSTO, 2013).

Para esta finalidade, foi utilizada a Classificação Hierárquica Descendente (CHD) e a análise de similitude como método de tratamento dos dados, o que possibilitou a identificação da frequência de cada palavra e sua conexão com as outras, além de auxiliar na análise do corpus textual (CAMARGO; JUSTO, 2013).

Ressalta-se que a interpretação e análise dos dados teve embasamento da literatura atual no que concerne à segurança do paciente e à gestão da qualidade em saúde.

Ressalta-se que o estudo seguiu os princípios éticos e legais que regem a pesquisa científica em seres humanos, preconizados na Resolução n 466/2012, do Conselho Nacional de Saúde, de forma a preservar o caráter voluntário dos participantes e o anonimato dos interlocutores. A pesquisa foi submetida para apreciação ética pelo Comitê de Ética da UFRN, após anuência da direção da ESUFRN, e foi aprovada a partir do Parecer nº 2.755.011, de 04 de julho de 2018. 


\section{RESULTADOS E DISCUSSÃo}

Foram convidados a participar das entrevistas 24 docentes, que ministraram módulos do Curso de Tecnologia em Gestão Hospitalar. Desses, a amostra obtida foi de 19 (79,2\%) docentes: $16(84,2)$ do sexo feminino e $3(15,8 \%)$ do sexo masculino. A média de idade dos sujeitos era de 43 anos (DP =9,8), com idade mínima de 30 e máxima de 57 anos.

Quanto à formação em nível superior, 12 (63,6\%) são formados em Enfermagem, 1 (5,2\%) em Ciências Biológicas, 1 (5,2\%) em Administração, 1 (5,2\%) em Odontologia, 1 (5,2\%) em Fisioterapia, 1 (5,2\%) em Educação Física, 1 (5,2\%) em Ciências Econômicas e 1 (5,2\%) em Psicologia. Quanto à maior titulação, 15 (78,9\%) são doutores e 4 (21,1\%) são mestres.

Desses sujeitos da pesquisa, $13(68,4 \%)$ não tiveram discussão ou capacitação durante sua formação profissional que abordasse a temática segurança do paciente, 2 (8,0\%) tiveram em cursos, 2 (8,0\%) em sua formação profissional, 2 (8,0\%) em mestrado, 2 (8,0\%) em doutorado, 1 (4,0\%) em especialização, 1 (4,0\%) em atuação profissional, 1 (4,0\%) em grupos de pesquisa e 1 (4,0\%) em congressos. O somatório é maior que 100,0\% devido aos sujeitos da pesquisa relatarem ter discussão ou capacitação acerca da temática em mais de um âmbito.

Quanto aos discentes, foram convidados a participar da pesquisa 22 concluintes do Curso Tecnológico em Gestão Hospitalar. Desses, a amostra foi de 12 (54,5\%) discentes: 9 $(75,0 \%)$ do sexo feminino e $3(25,0 \%)$ do sexo masculino. A média de idade era de 29 anos (DP =9,0), com idade mínima de 20 e máxima de 47 anos.

Dos discentes, 8 (66,7\%) possuíam formação profissional, sendo $4(33,4 \%)$ Técnicos em Enfermagem, 1 (8,3\%) Técnico em Radiologia, 1 (8,3\%) graduado em Fisioterapia, 1 (8,3\%) graduado em Enfermagem e 1 (8,3\%) graduado em Saúde Coletiva.

A análise do corpus proveniente da transcrição das 31 entrevistas semiestruturadas denotou 6.449 ocorrências de palavras, apresentadas em 706 formas distintas. Por meio da Classificação Hierárquica Descendente foram analisados 144 segmentos de texto, retendo $78,26 \%$ do total para elucidação das classes. Visualiza-se, a seguir, o dendrograma que denota as seis classes advindas das partições de conteúdo (figura 1). 
Figura 1. Dendrograma do corpus textual analisado

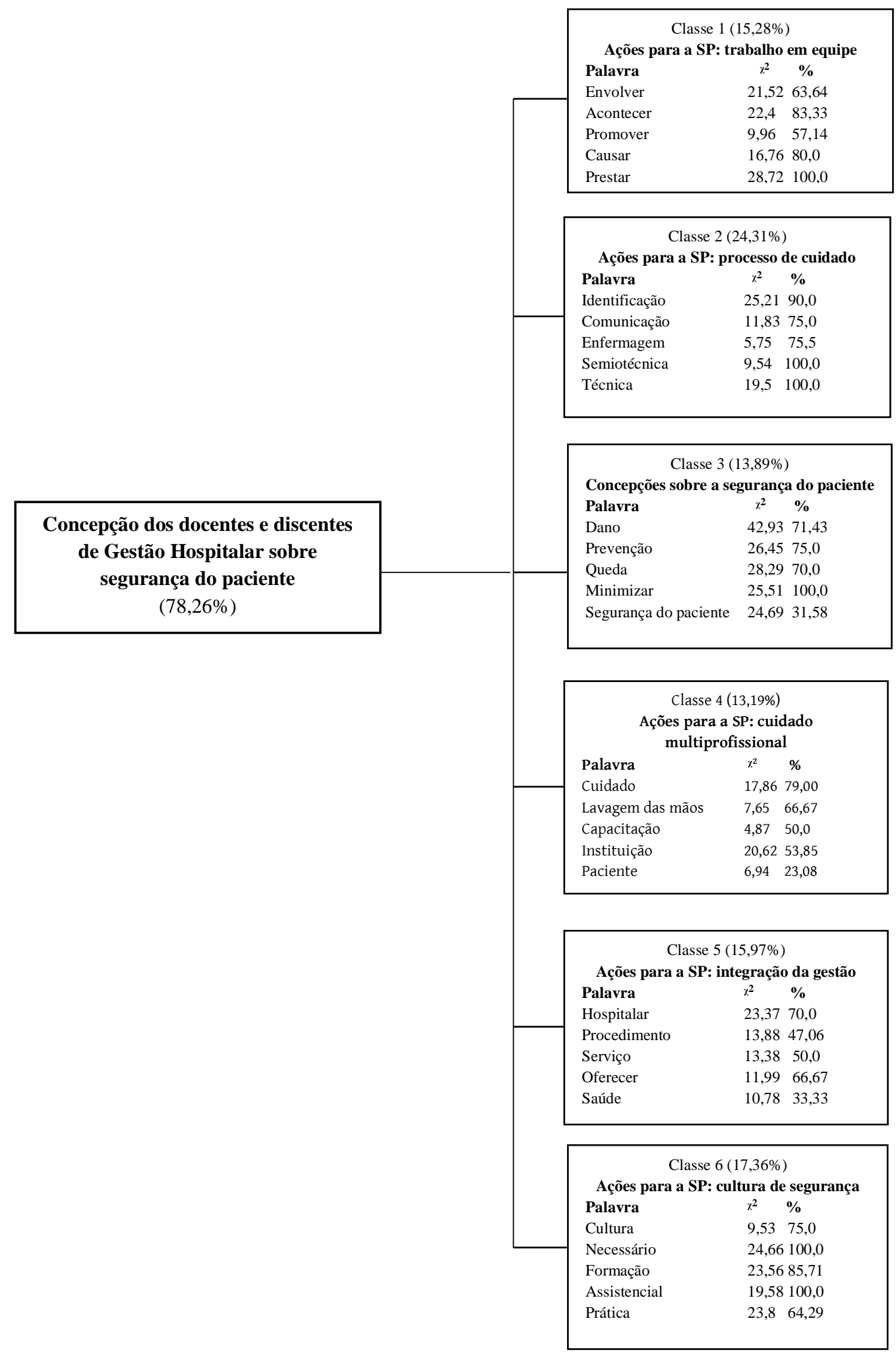

Fonte: Elaboração Própria. 
A classe 1, nominada "Ações para a SP: trabalho em equipe", representou 15,3\% dos segmentos de texto analisados no âmbito do corpus. As palavras envolver, acontecer, promover, causar e prestar denotam atitudes necessárias para que seja implementada, de fato, a segurança do paciente, o que pode ser observado na fala: Então uma forma de promover segurança do paciente é ter um ambiente de trabalho e um processo de trabalho que cause a menor possibilidade de adoecimento para a pessoa que preste o cuidado (Docente 09).

Desse modo, compreende-se o trabalho em equipe como condição fundamental a um processo de trabalho que promova a segurança do paciente. Sobre isto, sabe-se que desde os primórdios da Antiguidade, o trabalho em equipe se configura como atividade coletiva em prol de algo. O trabalho em equipe surge da necessidade de estabelecer objetivos e metas em comum com um plano de trabalho bem definido, por meio do qual se desenvolvam o crescimento individual e do grupo e o cuidado centrado no usuário e na comunidade envolvidos (SILVEIRA; SENA; OLIVEIRA, 2011).

$\mathrm{Na}$ área da saúde, vê-se a necessidade de integração entre os profissionais da saúde para garantir a excelência no cuidado seguro, uma vez que essa deve ser uma ação em conjunto, tanto dos gestores, que estão na área administrativa, quanto dos profissionais, que estão na assistência.

A classe 2, "Ações para a SP: processo de cuidado" totalizou 24,3\% do corpus analisado. As principais palavras dessa classe foram: identificação, comunicação, enfermagem, semiotécnica e técnica. Apreende-se, por meio desse vocabulário, as ações do processo de trabalho dos profissionais de saúde integrado à segurança do paciente apresentadas na seguinte fala:

As ações associadas a essa temática eu acho que desde o processo de trabalho. 0 processo de trabalho ele está carregado dessa perspectiva. Acredito que ela seja fundante e passa através comunicação terapêutica, porque é através do lidar, de uma palavra, passa pela questão de procedimentos técnicos, fazê-los corretamente, respeitar o paciente (Docente 07). 
O processo de trabalho em busca do cuidado seguro, como visto nessa classe, aborda diferentes áreas, inclusive a gestão em saúde, no que deve buscar a qualidade no trabalho em equipe de saúde e nas intervenções, em geral, que possam ocorrer na assistência à saúde.

Sobre isso, é importante compreender as ações prioritárias do Programa Nacional de Segurança do Paciente (PNSP), que foram denominadas desafios globais e buscam reduzir a infecção associada ao cuidado em saúde, por meio da campanha de higienização das mãos, e promover uma cirurgia mais segura, pela adoção de uma lista de verificação antes, durante e após o ato cirúrgico, além de buscar a qualidade como prioridade em todas as ações de saúde (BRASIL, 2013).

Sobre este tema, a classe 4, denominada "Ações para a SP: cuidado multiprofissional", representou 13,2\% da análise textual realizada. Destacaram-se os vocábulos: cuidado, lavagem das mãos, capacitação, instituição e paciente, relevando, portanto, elementos do cuidado que os docentes e discentes destacaram como ações para que a segurança do paciente seja garantida. Dessa forma, pode ser evidenciado na fala:

As ações associadas a essa temática são cuidados, capacitação contínua das pessoas que fazem esse cuidado em que estão perto desse paciente para promover a segurança dele durante as intervenções que ele venha a assumir, educação em saúde, porque todo tempo você tem que retomar, reciclando e melhorando essa atuação (Docente 16).

Com o Programa Nacional de Segurança do Paciente (PNSP), instituído pela Portaria n 529/2013, com o objetivo geral de contribuir para a qualificação do cuidado em saúde em todos os estabelecimentos de saúde do território nacional, foram estabelecidos os seis protocolos de segurança do paciente, que são:

1. Identificação correta do paciente;

2. Prevenção de Lesão por Pressão;

3. Segurança na Prescrição, Uso e Administração de Medicamentos;

4. Cirurgia Segura;

5. Prática de Higiene das Mãos em Serviços de Saúde;

6. Prevenção de Quedas. 
Além de tais ações, o vocábulo da classe 5 permitiu a contextualização "Ações para a SP: integração da gestão", responsável por 16\% do corpus analisado. As palavras hospitalar, procedimento, serviço, oferecer e saúde denotam pontos que integram o cuidado seguro, entendendo a intersetorialidade da segurança do paciente. Desse modo, pode ser representado pela fala: Eu acho que essas ações perpassam por tudo que acontece com ele na unidade hospitalar ou numa unidade de saúde, então tudo, todos os setores devem trabalhar na segurança do paciente [...] (Docente 08).

O gestor do serviço de saúde deve dar prioridade à segurança do paciente para que essa seja uma prática aderida pelos profissionais, assim, Minuzz, Salum e Locks (2016) afirmam que

estudiosos apontam que promover a cultura de segurança demanda empenho e comprometimento de toda a instituição, porém com ênfase nos gestores, que devem atuar incentivando a comunicação e a notificação dos erros e fortalecendo a cultura não punitiva, proporcionando um clima de trabalho que prioriza a segurança do paciente (p.7).

Sobre isto, a classe 6, "Ações para a SP: cultura de segurança" do paciente, totalizou 17,4\% dos segmentos de texto analisado e foi construída a partir das seguintes palavras: cultura, necessário, formação, assistencial e prática. Denota, assim, pilares para se chegar, de fato, a um cuidado seguro, desde o conhecimento até a ação. Nesse sentido, cria-se uma cultura de segurança do paciente. Pode ser observado na fala de um docente:

Então, para ter realmente a segurança do paciente, é necessário primeiro ter essa cultura, que é exatamente compreender a importância dessas ações e mudar a minha, o meu modo de atuar. [...] então são essas ações, que vão desde a formação até realmente o momento prático, o momento assistencial, aquele próximo do paciente (Docente 10).

A cultura de segurança é definida como o produto de valores, atitudes, competências e padrões de comportamento individuais e de grupo, os quais determinam o compromisso, o estilo e a proficiência da administração de uma organização saudável e segura (BROWN et al. 2008). 
De maneira oposta à cultura punitiva, que, como o próprio nome diz, atribui toda a responsabilidade do evento adverso a determinado(s) profissional(is), julgando sua forma de atuação. Diante disso, é necessário que a cultura punitiva dê espaço à cultura de segurança, fazendo com que a notificação dos eventos adversos e análise de suas causas, sejam para aprendizagem contínua, objetivando o cuidado seguro.

Por fim, a classe 3, intitulada "Concepções sobre a segurança do paciente", representou $26,4 \%$ dos dados textuais analisados e as palavras mais citadas foram: dano, prevenção, queda, minimizar, segurança do paciente, que representam a construção do conceito de segurança do paciente. Desse modo, o seguinte trecho mostra a concepção a respeito do tema: Segurança do paciente são ações e condutas que evitem ou minimizem danos ao paciente, que podem ser prevenção de quedas e lesões por pressão, segurança nos procedimentos realizados com o paciente (Discente 7).

A SP surge da necessidade de evitar danos para os pacientes que estão nos serviços de saúde. Dessa forma, com os protocolos, busca-se que sejam desenvolvidas ações de cuidado seguro, como a identificação do paciente e a higienização das mãos. Paiva, Paiva e Berti (2010) entendem que

\begin{abstract}
A assistência à saúde envolve a articulação de conhecimentos e ações que se relacionam com serviços diferentes e tipos diversos de profissionais. A existência destes vários níveis de especialização e sua interdependência coloca as instituições de saúde como ambientes de alto risco para eventos adversos e incidentes. Definese eventos adversos como injúrias não intencionais decorrentes da atenção à saúde, não relacionadas à evolução natural da doença de base, que ocasionam lesões mensuráveis nos pacientes afetados e/ou prolongamento do tempo de internação e/ou óbito (p.288).
\end{abstract}

Assim, é de extrema importância sua discussão na formação em saúde. A presente pesquisa mostrou que a maioria dos docentes não apreendeu sobre a segurança do paciente na sua formação acadêmica, porque há um tempo não existia esse debate ou se existia era pouco difundido. Hoje, já se podem ver os frutos que o cuidado seguro pode trazer para a saúde dos usuários. Por isso, é profícuo que esse tema seja discutido na área acadêmica dos estudantes da saúde.

No ensino, o tema da segurança do paciente deve perpassar todo o currículo e enfocar especificidades de riscos e medidas preventivas de dano nos variados cenários de assistência 
à saúde. Deve ser desenvolvido por meio de ações de ensino-aprendizagem em que o aluno e o educador experienciem práticas significativas, que repercutam em uma atuação segura ao longo da formação e que se sustentem também na atuação profissional (URBANETTO; GERHARDT, 2013).

\section{CONSIDERAÇÕES FINAIS}

O presente estudo mostrou a concepção dos docentes e discentes de Gestão Hospitalar a respeito da segurança do paciente, que compreendem a importância do conceito e das ações para, assim, disseminar uma cultura de segurança. É possível observar, ainda, que a cultura de punição nas falas dos entrevistados é ausente.

Foram destacados fatores como: processo de trabalho e trabalho em equipe para a efetivação do cuidado seguro. Além disso, as entrevistas colaboraram para um pensamento de que a segurança do paciente deve perpassar por todos os profissionais, em especial o gestor, que planejará recursos para que essas ações aconteçam.

Dada à importância do assunto, torna-se necessário o desenvolvimento de capacitações para os gestores hospitalares sobre a SP, podendo, assim, contribuir para uma assistência à saúde mais segura.

\section{REFERÊNCIAS}

ANDRADE, L.E.L.; LOPES, J.M.; SOUZA FILHO, M.C.; VIEIRA JÚNIOR, R.F.; FARIAS, L.P.C.; SANTOS, C.C.M.; GAMA, Z.A.S. Cultura de Segurança do paciente em três hospitais brasileiros com diferentes tipos de gestão. Ciência e Saúde Coletiva, v. 23, n. 1 p. 161-172, 2018.

BALSANELLI, A.P.;JERICÓ, M.C. Os reflexos da gestão pela qualidade total em instituições hospitalares brasileiras. Acta Paulista Enfermagem, v. 18, n. 4, p. 397-402, 2005

BRASIL, Ministério da Saúde. Portaria nº. 529, de 1 de abril de 2013. Institui o Programa Nacional de Segurança do Paciente (PNSP). Diário Oficial da União 1 abr 2013. Disponível em: <http://bvsms.saude.gov.br/bvs/saudelegis/gm/2013/prt0529_01_04_2013.html>. Acesso em: 12 ago. 2019.

BROWN, C.; HOFER, T.; JOHAL, A.; THOMSON, R.; NICHOLL, J.; FRANKLIN, B.D.; LILFORD, R.J. An epistemology of patient safety research: a framework for study design and interpretation. Part 1. Conceptualising and 
developing interventions. Quality and Safety in Health Care., v. 17, n. 3, p. 158-162, 2008. Disponível em: <https://qualitysafety.bmj.com/content/qhc/17/3/158.full.pdf>. Acesso em: 12 ago. 2018.

CARVALHO, C.O.M.; SARDENBERG, C.; MATOS, A.C.C.; CENDOROGLO NETO, M.; SANTOS, B.F.C. Qualidade em Saúde: Conceitos, desafios e perspectivas. Brazilian Journal of Nephrology, v. 26, n. 4, p. 216-222, 2004.

GOMES, A.T.L.; FERREIRA JUNIOR, M.A.; SALVADOR, P.T.C.O.; BEZERRIL, M.S.; CHIAVONE, F.B.T.; SANTOS, V.E.P. Segurança do paciente em situação de emergência: percepções da equipe de enfermagem. Revista Brasileira de Enfermagem, v. 72, n. 3, p. 753-759, 2019.

KAWAMOTO, A.M.; OLIVEIRA, J.L.C.; TONINI, N.S.; NICOLA, A.L. Liderança e cultura de segurança do paciente: percepções de profissionais em um hospital universitário. Journal of Research: Fundamental Care Online, v. 8, n. 2, p. 4387-4398, 2016.

MINUZZ, A.P.; SALUM, N.C.; LOCKS, M.O.H. Avaliação da cultura de segurança do paciente em terapia intensiva na perspectiva da equipe de saúde. Texto e Contexto Enfermagem, v. 25, n. 2, e1610015, 2016.

NATIONAL PATIENT SAFETY FOUNDATION. Sem danos: acelerar a melhoria da segurança do paciente - quinze anos depois de To Err Is Human. Boston (US): The National Patient Safety Foundation. 2015. Disponível em: <https://proqualis.net/sites/proqualis.net/files/Sem\%20dano_\%20Acelerando\%20a\%20melhoria\%20da\%20seg uran\%C3\%A7a\%20do\%20paciente\%20quinze\%20anos\%20ap\%C3\%B3s\%20Errar\%20\%C3\%A9\%20humano.pdf>.

Acesso em: 16 jul. 2019.

PAIVA, M.C.M.S.; PAIVA, S.A.R.; BERTI, H.W. Eventos adversos: análise de um instrumento de notificação utilizado no gerenciamento de enfermagem. Revista da Escola de Enfermagem da USP, v. 44, n. 2, p. 287-294, 2010. Disponível em: <http://www.scielo.br/pdf/reeusp/v44n2/07.pdf>. Acesso em: 12 ago. 2019.

REIS, C.T. A cultura de segurança do paciente: validação de instrumento de mensuração para o contexto hospitalar brasileiro. 2013. Tese (Doutorado). Escola Nacional de Saúde Pública Sérgio Arouca - FIOCRUZ. Rio de Janeiro, 2013.

SILVA, J.P.; RODOVALHO, J.L. Concepção de avaliação da qualidade nos serviços de saúde. Tese (Doutorado). Pontifícia Universidade Católica de Goiás. Goiás, 2012.

SILVEIRA, M.R.; SENA, R.R.; OLIVEIRA, S.R. O processo de trabalho das equipes de saúde da família: implicações para a promoção da saúde. Revista Mineira de Enfermagem, v. 15, n. 2, p. 196-201, 2011. <http://www.reme.org.br/content/imagebank/pdf/v15n2a06.pdf>. Acesso em: 12 ago. 2019.

URBANETTO, J.S.; GERHARD, L. M. Segurança do paciente na tríade assistência ensino pesquisa. Revista Gaúcha de Enfermagem, v. 34, n. 3, 2013. Disponível em: <https://seer.ufrgs.br/RevistaGauchadeEnfermagem/article/view/43294>. Acesso em: 12 ago. 2019.

VITURI, D.W.; ÉVORA, Y.D.M. Total Quality Management and hospital nursing: an integrative literature review. Revista Brasileira de Enfermagem, v. 68, n. 5, p. 660-667, 2015.

$(\mathrm{cc}) \mathrm{Br}$

Este trabalho está licenciado com uma Licença Creative Commons - Atribuição 4.0 Internacional. 